\title{
Andere, Selbste, Grenzen. Momentaufnahme eines Diskurses zur globalen Migration
}

Anne Sophie Krossa (Sophie.Krossa@kh-mz.de) Catholic University of Applied Sciences Mainz, Germany

The social, society in a broad sense, can be demonstrated particularly well in relation to borders and frontiers, as the latter are the meeting site for the different as well as the similar, and the aspect of separation is inextricably linked to that of connection. How does globalization impact on assessments of selves and others, and how does this condense as border representations on various levels, for instance the national, the European and the global ones? To answer these questions, results of a discourse analysis will be presented. They are based on press commentaries that followed a very serious accident involving refugees off Lampedusa in October 2013 and on the reaction of Germany's then Minister of the Interior, H.-P. Friedrich ("Dublin II remains unchanged, to be sure"). Issues of particular interest are the following. How and in which forms are borders and frontiers represented discursively? Which underlying notions of others and corresponding selves manifest themselves as a consequence? Which functions are ascribed to borders and frontiers? And which unresolved ambivalences remain, as revealed by the discourse? The text is framed by preliminary thoughts derived from considering which potentials of sociation may be detected under the specific conditions.

\section{Keywords}

Globalization, Border/Frontier, Society, Conflict, Others, Selves, Migration, Discourse

An Grenze lässt sich Soziales, Gesellschaft im weiteren Sinne, besonders gut festmachen, denn an ihr trifft sich Differentes sowie Ähnliches, und der Aspekt der Trennung ist von dem der Verbindung nicht zu separieren. Wie wirken Globalisierungseinflüsse auf Einschätzungen von Selbsten und Anderen, und wie schlägt sich dies in Grenzrepräsentationen auf verschiedenen Ebenen nieder, beispielsweise der nationalen, der europäischen und der globalen? Dazu werden Ergebnisse einer Diskursanalyse auf der Grundlage von Pressekommentaren präsentiert, die in Folge eines schweren Flüchtlingsunglückes vor Lampedusa im Oktober 2013 und der Reaktion des damaligen Innenministers Friedrich („Dublin II bleibt unverändert, selbstverständlich“) erschienen. Dabei interessiert besonders: Wie und in welchen Formen werden Grenzen diskursiv repräsentiert? Welche Vorstellungen von Anderen und entsprechenden Selbsten zeigen sich dabei? Welche Funktionen werden Grenzen zugesprochen? Und schließlich: Welche Ambivalenzen, die offen bleiben, zeigt der Diskurs auf? Der Text wird gerahmt von ersten Ableitungen zur Frage, welche Vergesellschaftungspotentiale unter den spezifischen Bedingungen erkennbar sind.

\section{Schlüsselwörter}

Globalisierung, Grenze, Gesellschaft, Konflikt, Andere, Selbste, Migration, Diskurs 
An Grenze lässt sich Soziales, Gesellschaft im weiteren Sinne, besonders gut festmachen. Bereits seit Georg Simmel wissen wir, dass Vergesellschaftung einer Kombination von Übereinstimmung und Differenz bedarf - in Simmels Begrifflichkeit genauer: Antagonismus im Sinne von sozial relevanter, gleichsam aktivierter Differenz. An Grenze trifft sich Differentes sowie Ähnliches, und der Aspekt der Trennung ist von dem der Verbindung nicht zu separieren ${ }^{1}$. Auf dieser Grundlage eröffnet sich mit Grenze ein flexibler Raum des Sozialen mit kreativen und destruktiven, ambivalenten und oft paradoxen Potentialen. Dies betrifft insbesondere Definitionen von Selbsten und Anderen. Dass die Festlegung des Anderen, ,Fremden', ein kontingenter Definitionsakt ist, der üblicherweise in Pluralität mündet, also mehrere, situationsabhängig unterschiedliche relevante Andere hervorbringt, ist grundlegend anerkannt. Prinzipiell analog ist auch das ihnen gegenübergestellte Selbst als kontingent zu begreifen, das sich in verschiedenen Situationen je andersartig aus gleichsam dialektischen Konstellationen konstituiert - deshalb auch hier der Plural ,Selbste'. Wie also wirken spezifischer Globalisierungseinflüsse auf Einschätzungen von Selbsten und Anderen, und wie schlägt sich dies in Grenzrepräsentationen auf verschiedenen Ebenen nieder, beispielsweise der nationalen, der europäischen und der globalen?

Zur Annäherung an diese Fragen werden im Folgenden Ergebnisse einer Diskursanalyse vorgestellt und mit einigen interdisziplinären theoretischen Aspekten von Grenze verbunden. Der zur Analyse ausgewählte Diskurs fokussiert Repräsentationen von Grenzen zwischen Europa und dem Globalen, letzteres hier vertreten durch $A f r i k a^{2}$, anhand europäisch-globaler, innereuropäischer und innerdeutscher Grenzziehungen.

Dazu wird anhand der sogenannten Dublin-Verordnung eine Zuspitzung der umfassenden Diskussion um globale Flüchtlinge in Europa in den Blick genommen. Rechtlich bestimmt seit dem 1.1.2014 die Verordnung (EU) Nr. 604/2013 (Dublin III), dass von Menschen aus sogenannten Drittstaaten ein Asylantrag nur in demjenigen europäischen Land gestellt werden darf, in das sie als erstes eingereist sind. Anhand von Pressebeiträgen wird der diesbezügliche Diskurs betrachtet, der sich im kurzen Zeitraum vom 8. bis 11. Oktober 2013, als Reaktion auf Kommentare des damaligen deutschen Innenministers Hans-Peter Friedrich entwickelte ${ }^{3}$. Zum Anlass eines - zum damaligen Zeitpunkt - außergewöhnlich schweren Bootsunglückes am 3. Oktober 2013, bei dem vor der Küste Lampedusas fast 400 Flüchtlinge starben, äußerte Friedrich: „Dublin II bleibt unverändert, selbstverständlich“ (zit. nach Handelsblatt, 8.10.) ${ }^{4}$. Friedrichs Aussage rief eine Welle von vielfältigen und gleichzeitig spezifischen Reaktionen hervor, die für die folgende Analyse als Grundlage dienen ${ }^{5}$. Heute, im November 2015, liest sich dies zum einen wie ein Kommentar zur bekannten Chronologie der Ereignisse und fungiert somit als eine Grundlage, zum anderen bleiben die Konzepte bemerkenswert gültig, ebenso wie Muster des Umgangs mit ihnen.

\footnotetext{
${ }^{1}$ Im Englischen wird dies begrifflich getrennt, in frontier im primär abgrenzenden Sinne und border mit Schwerpunkt bei Angrenzung (siehe auch zur Semantik des EU-Rechts Delanty und Rumford 2005, S. 31). Eigmüller und Vobruba (2006, S. 9) nennen außerdem boundary, bounds und limits.

${ }^{2}$ Erster Ausgangspunkt diesbezüglich ist die Annahme, dass der „'Mittelmeerraum' als geopolitisches Konzept“ imaginiert wird, welches für Europa "die Grundlage für die Konstruktion des geopolitischen ,Anderen' - Afrika - ist“ (Strüver 2008 , S. 66) und als Folie für entsprechende Selbstkonstruktionen dient.

${ }^{3}$ Ich danke Kora Hoffmann und Diana Steegers für ihre tiefgehenden Annäherungen an das Thema, die zu sehr hilfreichen Vorbereitungen der hier zugrunde gelegten Diskursanalyse geführt haben.

${ }^{4}$ Dublin III trat zum 1.1.2014 in Kraft. Die Vorgängerregelung, Dublin II, auf die sich Friedrich noch bezieht, galt seit März 2003 und war im hier interessierenden Punkt inhaltsgleich.

${ }^{5}$ Die anhaltende Relevanz dieses Ereignisses und entsprechender Diskurse beweist sich aktuell - im April 2015.

Nach einem weiteren, noch größeren Flüchtlingsdrama nach dem gleichen Muster dient das hier analysierte als immer wieder angeführter Referenzpunkt.
} 
Forschungsleitend sind für diesen Text die folgenden Fragen: Wie und in welchen Formen werden Grenzen diskursiv repräsentiert? Welche Vorstellungen von Anderen und entsprechenden Selbsten zeigen sich dabei? Welche Funktionen werden Grenzen zugesprochen? Und schließlich: Welche Ambivalenzen, die offen bleiben, zeigt der Diskurs auf?

Zur Annäherung an Antworten auf diese Fragen ist der Text in vier Bereiche unterteilt. Im ersten Abschnitt geht es um die Frage, inwiefern Grenze als Linie oder als Raum interpretiert wird. Die beiden Hauptbezugsebenen - das Europäische und das Globale - scheinen dabei entgegengesetzten Imperativen zu unterliegen: Während im EU-Rahmen Grenzen aufgehoben werden und nur noch ein gemeinsamer Raum bestehen soll ${ }^{6}$, ist dem Globalen idealtypisch eine Grenze in Linienform entgegengesetzt. Zusammengefasst lautet die Schlussfolgerung dazu, dass sowohl Linie als auch Raum in verschiedenen Kontexten erkennbar sind und es gute Gründe gibt, beide Konzepte nicht als sich gegenseitig ausschließend aufzufassen. Zweitens werden Interpretationen von Anderen und ihre Implikationen für Selbstkonstituierungen betrachtet. Dabei zeigen sich ebenfalls Verschiebungen, die u.a. darauf beruhen, dass in unterschiedlichen Perspektiven und Situationen unterschiedliche Grenzen aktiviert werden, so dass verschiedene Andere jeweils Selbstrelevant werden. Außerdem wird deutlich, dass klare Selbst- und Fremdgrenzen nicht ohne weiteres bestehen. Im dritten Punkt werden die beiden grenzkonstitutiven Aspekte Abgrenzung und Verbindung betrachtet. Auch dabei wird weniger eine eindimensionale Entscheidung denn eine Differenzierung vorgenommen, beispielsweise in Richtung Relationierung und Ausgleich bzw. Interpretationen von Fairness. Schließlich wird im vierten Teil untersucht, wie sich die zunehmend offensichtlich werdende Kontingenz von Grenzen auf den Umgang mit innen auswirkt und welche Ambivalenzen in diesem Diskurs zu globaler Migration im europäischen Kontext bestehen bleiben. Der Text wird lose gerahmt von ersten Ableitungen zur Frage, welche Vergesellschaftungspotentiale unter den geschilderten Bedingungen erkennbar sind.

\section{Linie und Raum}

Analytisch liegt der Fokus fraglos auf einer räumlichen Konzeption von Grenze im Gegensatz zu einer linearen. Dies steht in direktem Zusammenhang mit einer zunehmenden Infragestellung der Eindeutigkeit und Stabilität von zugrunde liegenden räumlichen Einheiten, die auch in der Soziologie traditionell vor allem als Nationalstaaten gesehen wurden ${ }^{7}$, denn Grenze kann nur dann als Linie funktionieren, wenn die Einheiten, die sie voneinander abtrennt, sogenannte Container-Form haben, also essentialistisch interpretiert sind. Die Tatsache, dass soziale Einheiten nicht homogen sind, ihre Beziehungen typisch übergreifen und so fortlaufend Vermischungen und Hybridisierungen produzieren, verhindert jedoch zum einen nicht das Fortführen dieser Interpretationslinie, auch in indirekteren Formen ${ }^{8}$. Zum anderen - und das ist im Sinne des Thomas-Theorems sicher interessanter - schmälert sie ebenfalls nicht unbedingt die Vorstellung von Grenzen als klar abgrenzenden Linien; manche vermuten im Gegenteil, ein zunehmendes Kontingenzbewusstsein befördere solche Interpretationen sogar ${ }^{9}$.

\footnotetext{
${ }^{6}$ Generell, aber auch zu den ambivalenten Aspekten siehe beispielsweise Bös 2001; Vobruba 2005; Bach 2009.

7 Entsprechende Fragen - z.B. ,Ist der Nationalstaat in Auflösung begriffen?' - wurden und werden umfassend besprochen (Krossa 2013; Schroer 2012; Ray 2007).

${ }^{8}$ So z.B. in der Annahme: „,border theory seeks to show how identity and other aspects of cultural life act as barriers or bridges between and among people, with particular emphasis on ethnic, racial and gender identity" (Wilson 2012, S. 79)

9 "Gerade vor dem Hintergrund sich auflösender Grenzen scheint das Containermodell erneut an Attraktivität zu gewinnen. Selbst wenn sich diese Schließungsszenarien und Abschottungsstrategien als Illusion erweisen, so sind sie doch überaus gebräuchliche und wirkungsmächtige Illusionen" Schroer (2012, S. 179). Zu vermuten ist jedoch, dass diese Illusion weitgehend auch als solche begriffen wird, denn : Die Vorstellung, an einem abgeschlossenem, abschließbaren
} 
Auf beide Formen - Linie und Raum - wird sowohl in klassischen als auch in neueren Perspektiven immer wieder Bezug genommen. Trotz der verbreiteten Referenz soziologischer Klassiker auf Nationalstaaten als gleichsam fixierte Räume von Gesellschaft wird bereits in frühen soziologischen Perspektiven der Grenzbegriff zumindest in Aspekten relativ offen angegangen ${ }^{10}$. Zwei soziologische Klassiker sollen dazu knapp erwähnt sein: Émile Durkheim und Georg Simmel.

Émile Durkheim beschäftigt sich über Gesellschaftsformen indirekt mit den beiden Idealtypen von Grenze. Einfache Gesellschaften, die er als weitgehend homogen und segmentär differenziert vorstellt, weisen seiner Meinung nach prinzipiell einen Containercharakter auf. Im Grenzbezug ist dabei besonders sein Hinweis auf das Konzept Niemandsland interessant, das zwei Gesellschaften dieses Typs trennt und gleichsam eine breite Linie darstellt. Der eigentliche Raumcharakter im Sinne eines sozialen Potentials wird dabei also aufgehoben, weil in dieser Zone keinerlei sozialer Austausch bzw. Wechselwirkungen mit potentiellen Vergesellschaftungsfolgen stattfinden (dürfen). Durkheims zweite Gesellschaftsform folgt dem Prinzip der funktionalen Differenzierung, die mit einer zunehmenden Öffnung sozialer Räume einhergeht und damit den Liniencharakter ihrer Grenzen aufweicht ${ }^{11}$. In Durkheims sozial-evolutionärer Perspektive erscheint die linearabgrenzende Form von Grenze als ein Übergangsstadium, möglicherweise sogar als Bedingung sine qua non für sich öffnende und dann Raumform annehmende Grenzen ${ }^{12}$.

Mit Georg Simmel wird in mehrfacher Hinsicht ein Perspektivenwechsel vorgenommen (Simmel 1992; auch Löw 2012 und Eigmüller 2006), wobei sein Ausgangspunkt die Annahme ist, Grenze sei eine soziologische Tatsache ${ }^{13}$. Hier interessiert vor allem, dass Simmel Abgrenzung nicht als gesetzten Ausgangspunkt heranzieht, sie nicht einmal in den Vordergrund stellt. Stattdessen fragt er generell nach Wechselwirkungspotentialen, speziell unter Bedingungen von Konflikt und sogar Krieg, und transzendiert damit ein reines Prinzip der Abgrenzung von vornherein: „Jede Grenze ist ein seelisches, näher: ein soziologisches Geschehen; aber durch dessen Investierung in einer Linie im Raum gewinnt das Gegenseitigkeitsverhältnis nach seinen positiven und negativen Seiten eine Klarheit und Sicherheit - freilich oft auch eine Erstarrung - die ihm versagt zu bleiben pflegt, solange das Sich-treffen und Sich-scheiden der Kräfte und Rechte noch nicht in eine sinnliche Gestaltung projiziert ist und deshalb immer sozusagen im status nascens verharrt" (Simmel 1992, S. 699). Auch, wenn es vordergründig so erscheint, als interpretierten Simmel und Durkheim die Bedeutung von Grenze für Ordnung und Orientierung ähnlich (Schroer 2012, S. 69), liegt doch bei Simmel das Augenmerk an ganz anderer Stelle: Er fokussiert gerade nicht die vermeintliche Stabilität, welche klar abgrenzende, lineare Grenzen nach innen herstellen könnte, sondern bezieht sich von vornherein auf etwas Übergreifendes und dessen Qualitäten. Denn Grenze wird, so Simmel, „doch zu einer lebendigen Energie, die jene auseinanderdrängt und sie (gleichzeitig, d.A.) nicht aus ihrer Einheit herauslässt" (Simmel 1992, S. 697). Die ,Linie im Raum' bedeutet bei ihm also: Es gibt einen gemeinsamen Raum der beiden Einheiten, die eben von vornherein nur be-

Ort zu leben, wird überall erfahrbar fiktiv“ (Beck 1997, S. 132; zit. nach Schroer 2012, S. 200). Dieser Aspekt wird im vierten Teil genauer besprochen.

${ }^{10}$ Allgemeiner zur Etymologie des Begriffs siehe Eigmüller 2008; Eigmüller und Vobruba 2006. Zur Geschichte von Grenze siehe z.B. Medick 2006.

11 „Die Gesellschaft hört auf, als das alleinige Ganze zu erscheinen, um Teil eines viel größeren Ganzen zu werden, mit unscharfen Grenzen, die fähig sind, unendlich nachzugeben (...). Anscheinend löst sich somit das Band, das zunächst das Denken an bestimmte kollektive Individualitäten gebunden hatte, immer mehr" (Durkheim 1984, S. 594), so dass schließlich „,soziale und räumliche Nähe nicht mehr länger zusammenfallen“(Schroer 2012, S. 55).

12 Parsons bestätigt, es sei „clear, that no society could attain what we will call the 'advanced primitive' level of societal evolution without developing relatively clear-cut boundedness" (Parsons 1966, S. 39).

13 „Die Grenze ist nicht eine räumliche Tatsache mit soziologischen Wirkungen, sondern eine soziologische Tatsache, die sich räumlich formt" (Simmel 1992, S. 697). 
dingt als solche, also als in sich geschlossen, angesehen werden können. Die Linie ist ihm demnach insofern ein Ordnungsinstrument, als dass sie beide Seiten in direkte Beziehung zueinander setzt und diese Beziehung in irgendeiner Weise klärt. Auch lässt sich das an Simmels Bezug auf das sogenannte Niemandsland nachzeichnen. Für Simmel ist dies zunächst „der leere Raum als leerer (...), ein wüster Landstrich (...), Raum (..) als reine Distanz, als qualitätslose Ausdehnung" (Simmel 1992, S. 785) ${ }^{14}$. Doch er erweitert das Konzept sogleich: „Einen ganz andren Sinn gewinnt die Neutralität des unbewohnten Raumes, indem sie ihn zu positiven Diensten befähigt: seine Funktion, die bisher eine trennende war, kann auch eine verbindende werden. Begegnungen von Personen, die auf dem Gebiet der einen oder dem der anderen untunlich wären, können manchmal doch auf neutralem Gebiet stattfinden“ (ebd., S. 787) - womit dieser Raum von beiden Seiten zur Verbindung nutzbar ${ }^{15}$ und zum potentiellen „Träger und Ausdruck soziologischer Wechselwirkung" (Simmel 1992, S. 790) wird ${ }^{16}$. Damit erfahren sowohl Linie als auch Raum bei Simmel grundlegende und bereichernde Reinterpretationen.

Noch expliziter finden sich konzeptuelle Öffnungen zu Grenze als Raum in einer Reihe aktueller soziologischer Perspektiven, die prinzipiell annehmen, dass Grenze „nicht in erster Linie eine Trennung (ist), die Räume separiert, sondern eine, die sie differenziert" (Rigo 2006, S. 170). Ihre gemeinsamen Ausgangspunkte sind: Erstens ist Grenze nicht lediglich auf ein klar geordnetes Hin-und-Her zwischen verschiedenen, schlicht als entgegengesetzt zu verstehenden Elementen zu reduzieren, sondern basiert auf dem Prinzip ihrer Relationierung ${ }^{17}$, was zu Kontingenzen führt. Dies impliziert, zweitens, dass Grenze nur als Prozess gedacht werden kann, also als zeitlich fortlaufend, auf Kombinationen von Verstetigung und Wandel basierend. Dieser in verschiedenartigen Formen stattfindende Relationierungsprozess hat in der Regel raumbildende Konsequenzen, und zwar sowohl im territorialen bzw. örtlichen als auch im abstrakten räumlichen Sinne, beispielsweise in Form sich entwickelnder, gemeinsamer Institutionen. Eine Folge ist, drittens, dass Grenze nicht lediglich als Ort des angrenzenden Fremden angesehen werden kann, sondern gleichzeitiger Ort der Fremd- und der Selbst-(De)konstruktion ist.

Dazu gibt einige konkrete Vorschläge im Sinne von Grenzzonen, die entsprechend ausdrücklich nicht den Kriterien des Niemandslandes entsprechen. Im Gegensatz zu dessen Prinzip des Herausnehmens von Antagonismus und Ambiguität bzw. letztlich vom Sozialen schlechthin wird eine räumliche, prinzipiell offen konzipierte Zone mit der Möglichkeit von gemeinsamer, Bedeutungs- und Sinnherstellung' (Donnan und Wilson 1999, S. 64) explizit gemacht. Dazu schlägt Saskia Sassen beispielsweise den Begriff analytic borderlands vor, als "spaces that are constituted in terms of discontinuities; in them discontinuities are given a terrain rather than reduced to a dividing line" $(2013$, S. 211). Mit stärkerer Berücksichtigung des charakteristisch Konfliktuellen solcher Zonen entwickelt

\footnotetext{
${ }^{14}$ „Unter den vielfachen Fällen, in denen die Maxime: tu' mir nichts, ich tu' dir auch nichts - das Benehmen bestimmt, gibt es keinen reineren und anschaulicheren als den des wüsten Gebietes, das eine Gruppe um ihre Grenze legt; hier hat sich die innere Tendenz völlig in die Raumform hinein verkörpert" (Simmel 1992, S. 785).

15 „Ist eine solche Möglichkeit des Begegnens gegeben, ohne dass im übrigen einer von beiden seinen Standpunkt zu verlassen braucht, so ist damit (..) (eine) Objektivation und Differenzierung eingeleitet" (Simmel 1992, S. 789).

${ }^{16}$ Als Typus zwischen der Interpretation des Niemandslandes als Grenze einerseits und Raum andererseits lässt sich restricted zone verstehen. Am Beispiel einer Grenzzone an der tschechisch-österreichischen Grenze beschreiben Blaive und Lindenberger (2012, S. 98f.), wie eine hinsichtlich der Aktivitäten und Relationen der Bewohnerinnen und Bewohner des Raumes stark und gezielt begrenzende Politik das Soziale ganz spezifisch verengt und geprägt hat, bis hin zu ethnischer, sozialer und politischer Homogenisierung und entsprechenden sozialen Folgen. Ihre Analyse setzt 1925 an und folgt den Spuren dieser Weichenstellung bis ins Jahr 2008. Ein aktuelles Beispiel eines solchen Verengungsprozesses, auch angelehnt an Niemandslandprinzipien, sind Verabredungen im Krieg im Osten der Ukraine, wo versucht wird, ,neutrale Zonen' zwischen den Kämpfenden einzurichten. Diese sind allerdings nicht vollständig sozial entleert, aus innen wird gleichsam lediglich eine Funktion - hier die militärische - herausgetrennt.

${ }^{17}$ Zum Prinzip der Relationierung, mit dem über Grenze Verschiedenes über den einfachen Zusammenhang hinaus in Austausch gebracht wird, siehe auch Karafillidis 2010; zur Diskussion von ,substanzialistischen versus relationalen' Interpretationen in Bezug auf Raum siehe beispielhaft Schroer 2012 und Löw 2012.
} 
Suvendrini Perera den Begriff borderscape, mit "different temporalities and overlapping emplacements as well as emergent spatial organizations" (Perera 2007, S. 206f.) bilden. Bei Ludger Pries liegt der konzeptuelle und empirische Schwerpunkt bei transnationalen Räumen, die im Zug verschiedenartiger Migrationsbewegungen entstehen (z.B. 2008, 2010) ${ }^{18}$ und damit im weiteren Sinne als zum Teil extrem umfassende Grenzzonen interpretiert werden können.

Im hier herangezogenen empirischen Material sind sowohl Linien- als auch Rauminterpretationen erkennbar. Dem Muster der Linie folgend werden vor allem die Außengrenzen der EU charakterisiert, wenn auch nicht immer so explizit wie in Formulierungen wie „Festung Europa“ oder „Flüchtlinge vor den Toren Europas“ (Freitag-Kommentar, 9.10. $)^{19}$, die eine idealtypische Verteilung global/Linie und Europa/Raum sehr genau widerspiegeln. Dies wird durch den nur scheinbar relativierenden Begriff Tor noch verstärkt, weil sowohl der Wille als auch die Kontrolle über Öffnen und Schließen ja bereits in Zweifel stehen. Auch Begriffe wie Grenzsicherung oder Grenzschutz verweisen zunächst auf eine Linienvorstellung: „Konkrete Hilfe wollen die EU-Partner Italien nur bei der Grenzsicherung schicken“ (Der Spiegel, 8.10., ähnlich Die Welt 8.10.). Frontex wird als „europäische(r) Grenzschützer()“ und als „EU-Grenzschutztruppe“ (FAZ , 8.10. (a)) bezeichnet, und auch an Eurosur werden Erwartungen gestellt, die den Liniencharakter der Außengrenze herstellen bzw. schützen sollen: „Das System soll mit Hilfe von Satellitentechnik, Überwachungsdrohnen und einem intensiveren Informationsaustausch zwischen den Mitgliedsstaaten die Außengrenzen der EU sicherer machen“ (Euractiv, 8.10.). Auch hier findet sich eine direkte Gegenüberstellung von europäischem Raum und davon klar abgetrenntem Globalem. Dagegen spricht der Direktor der FRA (European Agency for Fundamental Rights) Kjaerum leicht relativierend von „unseren Grenzkontrollrechten“ (ORF.at, 8.10.).

Dem Linienprinzip entsprechend finden sich auch Containervorstellungen: „Philipp Mißfelder fordert, einen Ausbau der Grenzsicherung und der Entwicklungshilfe' (Die Welt, 9.10.), und ebenfalls wird verlangt, man solle dafür sorgen, dass potentielle Flüchtlinge „in ihren Ländern bleiben und sich aufgehoben fühlen“ (FAZ, 10.10.). Explizit wird dies regelmäßig zur semantischen Kurzform Afrika verdichtet, die das Gegenüber stellt (dazu genauer in Teil 2).

Es fällt jedoch auf, dass die globale Grenze als Linie nahezu immer als Aufforderung formuliert ist: Sie soll hergestellt, gesichert werden - was unmittelbar auf ihren prekären Status verweist. Reziprok stellen sich die Ergebnisse zum innereuropäischen Raum dar, der ebenfalls als direkte oder indirekte Aufforderung präsentiert wird, indem dauernd auf innereuropäische Grenzen als Problem verwiesen wird (dazu genauer unten). Dabei zeigen sich nationalstaatliche Grenzen aktiv: „Eine Aussprache (...) ergab aber auch Meinungsunterschiede - etwa zwischen Deutschland und Italien“ (FAZ, 8.10. (a), siehe auch Handelsblatt, 8.10.). Und besonders deutlich: „Deutschland zeigte Härte“ (ORF.at, 8.10.). Auch werden Grenzen zwischen einzelnen Staaten und europäischen Staatengruppen gezogen, sei es konkreter - „Deutschland gerät (...) zunehmend unter Druck, weil viele (...) von Berlin ein stärkeres Engagement verlangen, um die Mittelmeerländer zu entlasten“ (Die Welt, 8.10.) und „Die Randländer Europas wie Italien, aber auch Griechenland und Spanien, fühlen sich (...) seit Jahren überfordert" (Die Welt, 8.10.). - oder abstrakter: „Mikl-Leitner (sprach sich) für eine Entlastung der Hauptaufnahmeländer aus" (ORF.at, 8.10.). Dies spricht, jedenfalls auf Wahrnehmungsebene, nicht für einen völligen Bedeutungsverlust von Nationalstaaten, der Containerperspektive und klaren Linienziehungen.

\footnotetext{
${ }^{18}$ Konkreter zur Vorstellung von 'Europa als Borderland' siehe Balibar (2004).

${ }^{19}$ Im Textmaterial werden EU und Europa generell synonym verwendet.
} 
Repräsentationen vom Niemandslandkonzept ließen sich in verschiedenen Ausprägungen erkennen, v.a.mit örtlichen Bezügen auf das Mittelmeer. Einerseits wird es zwar als Aktionsraum betrachtet: „Die EU-Kommission schlug den Staaten einen Großeinsatz durch europäische Grenzschützer von Frontex vor, die das gesamte Mittelmeer von Zypern bis Spanien umfasst" (Die Welt, 8.10.). Andererseits aber nimmt es als Ort der vielen Toten Niemandslandcharakter an: „Vor der Küste der Insel setzten Taucher (... ) die Bergung weiterer Leichen aus dem (...) gesunkenen Flüchtlingsboot fort (...). Diese ziehen die Leichen mit Hilfe von Seilen und Kabeln an die Wasseroberfläche" (Süddeutsche, 9.10.). Und als Forderung: „Das Mittelmeer darf nicht weiter ein Massengrab für Flüchtlinge sein“ (FAZ. 10.10.). Örtlich lässt sich dieses Niemandsland im Sinne eines Ringes von Toten rund um Europa erweitern. Zeit online schätzt, dass an den Außengrenzen Europas jährlich zwischen 19.000 und 25.000 Menschen sterben: "Sie sterben in der Hand von Schlepperbanden, ertrinken im Mittelmeer oder ersticken in Lastwagen" (Zeit online, 9.10.). Mit den Menschen stirbt das Gesellschaftliche, nur ihr Tod wird noch verwaltet, Konflikt und Austausch finden am Ort selbst kaum Platz (beispielsweise auch durch das Verbot an italienische Fischer, Ertrinkenden zu helfen, siehe dazu Teil 3). In diesem Sinne besteht das nicht-soziale Element des Niemandslandes als Raum mit Liniencharakter, gemäß Durkheim. Gleichzeitig aber ist auch Simmels relationierendes, ordnungsschaffendes Verständnis dieses Raums erkennbar. Dass auch diese Ordnung konkreter, kritischer Analyse bedarf, ist selbstverständlich - und in diesem Sinne wird das Niemandsland als abstrakter Raum durchaus relevant, nämlich als Anlass für Diskurs und potentielle bzw. geforderte Verhandlungen mit dem Anderen. Als weitere Formen von Niemandsland lassen sich Lager in ihrer isolierenden, einkapselnden Wirkung (Süddeutsche, 9.10.) interpretieren, aber auch die Institution Verfahren (Zeit online, 9.10., Süddeutsche, 9.10.; dazu genauer in Teil 3).

\section{Selbste und Andere}

An Grenze finden gleichzeitig Fremd- und Selbstkonstitutionen statt, die kontingent sind und Definitionsprozessen unterliegen. Analog zur Unterscheidung Linie/Container und Raum/unbestimmtere ,Einheiten' lassen sich Interpretationen von Selbsten und Anderen idealtypisch in trennscharf und diffus unterscheiden.

Durch die analytische Öffnung des Grenzverständnisses hin zu Raum kommt es „zu einer Überlagerung von innen und außen, geschlossen und offen, eigen und fremd, Nähe und Ferne" (Schroer 2012, 78, Hervorhebungen d.A.). Damit wird zumindest diskussionswürdig, ob sozial funktionierender Ein- und Ausgrenzung anhaltend eine auch nur relativ klare Grundlage geboten ist. Denn zum einen ist - wie mit Simmel bereits gezeigt - das Potential von Grenzüberschreitung im Konzept der Grenze stets schon von vornherein enthalten. Dies zeigt spezifischer Reckwitz' Begriff limit im Sinne eines Grenzspekts an, der sich „bezieht (..) auf eine normative und asymmetrische Grenze, auf jene zwischen (...) dem Legitimen und der illegitimen Transgression, hinter der etwas wartet, was die Grenze in Richtung des Pathologischen wie Faszinierenden überschreitet" (Reckwitz 2008, S. 302). Zum anderen, und noch weitergehend, argumentiert Augé, dass „das Bild vom Anderen zunehmend verwischt und dadurch direkt oder indirekt die Krise des Andersseins ausgelöst wird“ (Augé 1995, S. 86) - sowie entsprechend eine Krise des Selbst. Das Balancieren des „Doppelgefühl(s) der Unterschiedlichkeit und der Übereinstimmung" (ebd., S. 92) wird zu einer nicht (mehr) ohne weiteres lösbaren Aufgabe, was in einem wachsenden Bewusstsein der nur relativen Andersheit des Anderen und des nur begrenzt stabilen Selbstseins mündet: "Disturbingly, the stranger is within us: we are our own strangers" (Kristeva 1988, zit. nach Gifford 2010, S. 28). Damit verändert sich sowohl das Innen-Außen- als auch das Selbst-Fremd-Schema grundlegend. 
Diese analytische Sichtweise ist jedoch, wie oben erläutert, sinnvoll durch Wahrnehmungen zu ergänzen, die in der Diskursanalyse auch besonders deutlich werden. Wie wird also das Andere wahrgenommen? Als lediglich ästhetisch anders, also primär potentiell kulturell bereichernd? Als zwar störend, aber dies nicht besonders schwerwiegend? Oder als essentiell anders und damit tendenziell bedrohlich? Wie werden entsprechend Abgrenzung bzw. Nähe repräsentiert? Welche Folgen hat das für Selbst-Repräsentationen? Einige Anregungen für diesen Aspekt der Diskussion werden nun in interdisziplinärer Perspektive vorgenommen und an die Diskursanalyse anschlussfähig gemacht.

Der Gegensatz zwischen essentialistischen und konstruktivistischen bzw. substanzialistischen und relationalen Interpretationen von Grenze wird in einer biologisch inspirierten, philosophischen Debatte anhand einer Gegenüberstellung von immunologischen und postimmunologischen Gesellschaftsinterpretationen aufgegriffen. Nach Roberto Esposito weist unsere Gegenwart einen immunologischen Charakter auf, dessen Grundmuster er in einer "Schutzreaktion gegenüber einem Risiko" $(2004$, S. 7) sieht: „Jemand oder etwas dringt in einen - einzelnen oder kollektiven - Körper ein und verändert, transformiert, verseucht ihn“ (ebd., S. 8). Charakteristische Ereignisse seien der „Kampf gegen das Aufflammen einer neuen Epidemie (...), die Verstärkung der Bollwerke gegen die illegale Einwanderung und die Strategien, die auf die Neutralisierung des neuesten Computervirus abzielen“ (ebd., S. 7). Von besonderem Interesse ist hier die Verortung, die er vornimmt: „(W)as konstant bleibt, ist der Ort, an dem die Bedrohung angesiedelt ist: und dies ist stets die Grenze zwischen Innen und Außen, Eigenem und Fremdem, Individuellem und Gemeinsamem" (ebd., S. 8). Hier ist Grenze stark als klar abgrenzende Linie gefasst, die auf in sich geschlossenen Einheiten basiert und entsprechende Formen von Grenzrepräsentationen erwarten lässt.

Byung-Chul Han nimmt eine direkte Gegenposition ein. Espositos Position zugrunde liege, so Han, „eine klare Trennung von Innen und Außen, von Freund und Feind oder von Eigenem und Fremdem (...). Angriff und Abwehr bestimmen das immunologische Handeln (...). Abgewehrt wird alles, was fremd ist. Der Gegenstand der Immunabwehr ist die Fremdheit als solche" (2014, S. 8; Hervorhebung d.A.). Dem entgegen stellt Han den Begriff einer postimmunologischen Gesellschaft, die sich gerade „durch das Verschwinden der Andersheit und Fremdheit aus(zeichnet) (...). (So) tritt an die Stelle der Andersheit die Differenz, die keine Immunreaktion hervorruft (...). Auch die Fremdheit entschärft sich zu einer Konsumformel. Das Fremde weicht dem Exotischen. Der Tourist bereist es" (ebd., S. 9; ohne Hervorhebungen des Originals). Und am konkreten Beispiel der Migrationsdiskussion schlussfolgert Han: „Einwanderer und Flüchtlinge werden eher als Belastung denn Bedrohung empfunden“ (S. 11).

Eine differenzierende Lesart dieses Arguments findet sich bei Polly Matzinger (2007), die im Kontext ihrer Krebsforschung eine erneuerte Unterscheidung ableitet: Statt, wie bislang in der medizinischen Forschung üblich, davon auszugehen, dass der Körper zwischen ,Selbst' und ,Fremd' unterscheidet und ,Fremd' abstößt - eine Annahme, die prinzipiell parallel zu der Espositos verläuft -, nimmt sie an, dass Körper innerhalb der Kategorie des Fremden zwischen freundlich (friendly) und gefährlich (dangerous) differenzieren. Damit wird die Aufmerksamkeit verschoben vom Differenten an sich zu spezifischen Qualitäten des Differenten, ohne allerdings, wie Han, den Begriff des Fremden vollständig zu ästhetisieren und ihn damit letztlich ebenso zu vereinseitigen, wie Esposito das mit umgekehrten Vorzeichen getan hat.

Diese biologisch-philosophisch gegründete Unterscheidung zwischen freundlich und gefährlich lässt sich parallel führen mit einer soziologischen, die Andreas Langenohl im Rahmen einer Studie zu Städtepartnerschaften vorgelegt hat (zusammenfassend Langenohl 2010), nämlich der Unterscheidung zwischen wertbezogenen essentiellen und 
ästhetischen Interpretationen kultureller Differenz. Er nimmt an, dass Differenz besonders dann unproblematisch - also besonders zugänglich für Vergesellschaftungsabläufe wird, wenn sie als ästhetische interpretiert wird, also gleichsam als Verkörperung von ,unterschiedlich, aber prinzipiell auf Augenhöhe, mit Bereitschaft zu Austausch“: „French wine, English biscuits, Italian sausages" (Langenohl 2015, S. 54). Damit wird die Grenze zwar prinzipiell reproduziert und aufrechterhalten, allerdings mit dem verbindenden Schwerpunkt border. Diesem Differenztyp gilt das Andere grundsätzlich als ,interessant'; Langenohl spricht mehrfach sogar von seiner celebration - was sich auch als Möglichkeit des genaueren Betrachtens und sogar Feierns des Eigenen anhand des nur graduell bzw. in weniger bedeutsamen Kategorien abweichenden und somit unbedrohlichen Anderen interpretieren ließe.

Demgegenüber folgt der zweite, sozial deutlich problematischer wirkende Differenztyp, dessen Unterschiede als essentielle wahrgenommen werden, vor allem dem Prinzip der Abgrenzung (frontier). Dies zeigt Langenohl anhand von Situationen, die im Rahmen von Kontakten einer gemeinsam reisenden Gruppe von Franzosen, Französinnen und Deutschen zu muslimischen Berliner Bürgerinnen und Bürgern entstanden (ebd., Chapter 8). Zusammengefasst ergibt sich das folgende Bild: Die Städtepartner aus Frankreich und Deutschland stehen den muslimischen Gastgebern gemeinsam befremdet gegenüber und interpretieren die Grenze zwischen ihnen als essentielle. Praktiken, die dies illustrieren, sind auffallend formales Verhalten wie bemüht diplomatische Formulierungen oder stark formales Einladungsverhalten zu gemeinsamem Essen, außerdem die explizit moderate Thematisierung, schwieriger' Aspekte, eine Schwerpunktlegung auf Sich-Erklären bei Bedachtheit auf Festhalten an der vermuteten Spezifität der sich gegenüberstehenden Positionen sowie bemühte Anerkennung der vermeintlichen besonderen - also grundlegend abweichenden - Kulturalität des Anderen. Dadurch entsteht eine Engführung des Austauschs, die sich beispielsweise bei Meinungsunterschieden nicht in offener Kontroverse mit dem vermeintlich essentiell Anderen ausdrückt, sondern sich stattdessen in einem lediglich innerhalb der deutsch-französischen 'Eigengruppe' stattfindenden, aufgeregten Gespräch manifestiert - also der Eigengruppe in dem Sinne, als dass sie die als lediglich ästhetisch anders interpretierten Anderen zusammenschließt. Entsprechend wird anhand der verschiedenen Praktiken der Distanzbetonung bzw. Distanzierung in diesem Beispiel eine Grenze ,Europäer/muslimische Migrantinnen und Migranten' (re)produziert ${ }^{20}$ - und nicht beispielsweise eine Grenze zwischen denen, die in Deutschland leben einerseits und denen, die in Frankreich leben andererseits.

Schließlich enthält Langenohls Beispiel den wichtigen Aspekt der Gegensatzkonstruktion in Form einer Simplifizierung des Anderen und dem Anspruch eines differenzierten Selbst, genauer: von positiv interpretierter ,Vielfalt' (Europa) einerseits und als problematisch betrachteter, abweichender Eindimensionalität' andererseits, nämlich den Verweis auf muslimische Migrantinnen und Migranten als Repräsentation des Globalen in seiner unterstellten unberechenbaren Fremdheit: "what distinguishes Europeans from migrants is that the latter are seen as exemplars of their culturality while the former are seen as cultivated in their diversity" (ebd., S. 216).

Damit wird in beiden Perspektiven, die den Aspekt der Fremdheit differenzieren, also sowohl in der biologischen-medizinischen Matzingers als auch in der soziologischen Langenohls, die Notwendigkeit von Einschätzungsleistungen gegenüber dem Anderen deutlich, die fraglos vielfältig, kontingent, riskant und potentiell folgenreich für Grenzrepräsentationen sind.

\footnotetext{
20 "The problem consisted (..) not so much in a stereotype about other local cultures, but in the conviction that others have a serious global culture" (Langenohl 2014, S. 219), eine, deren Abgrenzung als praktisch unüberwindbar konstruiert wurde.
} 
Im Textmaterial zeigt sich dazu grundsätzlich, dass eine Tendenz zu quantitativer Erweiterung besteht: Statt einer Trennlinie zwischen einem Selbst und einem Anderen bestehen mehrere Selbste, Andere und Grenzen, die in unterschiedlichen Zusammenhängen aktiviert werden. Außerdem werden sie - situativ differenziert - mit qualitativ unterschiedlichen Differenzkennzeichnungen versehen, was prinzipiell den zuletzt beschriebenen Ansätzen entspricht, die das Andere weder als pauschal gefährlich noch als rein ästhetisch betrachten, sondern innerhalb seiner Kategorie differenzieren. Gleichzeitig finden sich jedoch ebenso immer wieder erhebliche Vereinfachungen in Wahrnehmung und Repräsentation, so dass die erhebliche Flexibilität auch zu paradoxen Formen führt.

Eine erste Distanzierung zum Anderen verläuft über Verkürzungen. Die bereits in der Einleitung erwähnte Vermutung, Afrika sei für Europa ein relevantes kollektives Anderes, wird vom Korpus wiederholt bestätigt. Erstens findet sich Afrika immer wieder und generell unreflektiert als semantische Verkürzung, die scheinbar ganz selbstverständlich das Andere konstituiert. Beispiele sind: 'Flüchtlingsstrom aus Afrika' (Martin Schulz, zitiert im Spiegel, 8.10.), 'Flüchtlingsandrang aus Afrika' (Die Welt, 8.10.), oder „The Mediterranean represents the Africa-Europe border, not the Africa-Italy border" (The Guardian, 8.10.). Entsprechend selten sind Differenzierungen des Afrika-Anderen, so Formulierungen wie 'nordafrikanische Länder' (Kölnische Rundschau, 8.10.). Bezüge auf einzelne Länder kommen fast nie vor; die einzige Ausnahme ist in diesem Textkorpus die namentliche Nennung Libyens und Tunesiens durch ORF.at (8.10). Im Gegensatz dazu wird die europäische Selbst-Seite dauernd und bis in Details differenziert, wie im Textverlauf immer wieder illustriert wird.

Die analoge semantische Figur des Anderen auf der individuellen Ebene, die in praktisch jedem Beitrag vorkommt, ist die des (afrikanischen) Flüchtlings. Doch auch dabei handelt es sich fraglos um eine künstliche Figur, die nur sehr selten differenzierend betrachtet wird. Während Herkunft, Fluchtgeschichte etc. nicht als individualisierende Aspekte genannt werden, wird eine Trennlinie zwischen bereits anwesenden und erst ankommenden Flüchtlingen erwähnt: „Auf Lampedusa haben Flüchtlinge gegen ihre Unterbringung in einem Auffanglager protestiert. Sie (...) versuchten, Busse mit Neuankömmlingen auf dem Weg in das überfüllte Lager aufzuhalten" (Süddeutsche, 9.10.). Ebenfalls nur sehr indirekt wird differenziert zwischen der Gruppe der bereits in Europa außerhalb der Lager und der noch in Gemeinschaftsunterkünften lebenden Flüchtlinge: „Viele Flüchtlinge sterben auf dem Weg nach Europa. Schaffen sie es doch, leben sie oft verarmt und unsicher" (Zeit online, 9.10.).

Ein zweiter Distanzierungsschritt lässt sich darin erkennen, dass bei 'Afrika selbst' das Problem verortet wird, und 'wir' entsprechend nur assistieren könnten: „Mit den afrikanischen Staaten müsse die EU darüber reden, wie sich die Fluchtgründe beseitigen ließen“ (Handelsblatt, 8.10.), mit dem Ziel, dass die potentiellen Flüchtlinge in „ihren Ländern aufgehoben bleiben und sich aufgehoben fühlen“ (FAZ, 10.10.) ${ }^{21}$. Das Selbst und das Andere werden auf diese Weise so trennscharf wie möglich konstruiert.

Drittens schließlich, und besonders massiv in der Verfremdungs- und Distanzierungswirkung, wird immer wieder auf Kriminalität, Illegalität etc. angespielt oder auch direkt verwiesen, sei es affirmativ oder nicht, so beispielsweise mit den Formulierungen 'illegale Einwanderung' (Spiegel, 8.10.) oder 'Asylmissbrauch' (Spiegel, 8.10.), bzw. wie in diesem Beispiel des Guardian: „Friedrich said that most of those trying to cross the Mediterranean were 'economic' migrants rather than political refugees and they were seeking better social security than they would receive at home" (8.10.). Somit wird die Grenze

\footnotetext{
${ }^{21}$ Umgekehrt wird die Verantwortung bzw. Schuld im folgenden Zitat bei der EU verortet, was allerdings ein Einzelfall ist: „,'Die Schleuserkriminalität ist eine Folge der für Flüchtlinge verschlossenen Grenze', kritisiert Pro Asyl“ (ORF.at, 8.10.).
} 
besonders wichtig und lässt sich als legitim darstellen: „Eurosur soll die Kommunikation zwischen den Staaten verbessern und so helfen, illegale Einwanderung zu erkennen sowie die grenzüberschreitende Kriminalität zu bekämpfen“ (Handelsblatt, 8.10.). Auf einer Mikroebene spiegelt sich dieses Verdachtsmuster in massiven Handlungsforderungen wider, so etwa in einem Aktionsplan der EU-Kommission, die „Zweckehen (..) bekämpfen und die Wohnsitze der Einwanderer leichter (..) ermitteln“ (Spiegel, 8.10.) will. Hier wird deutlich, wie stark der substantielle Verdacht ins Private reicht, 'fremd' pauschal als 'gefährlich' eingeschätzt wird, analog zu den Thesen der immunologischen Gesellschaft. Auf der individuellen Ebene ist der kriminalisierte Andere die/der Illegale, vor allem aber der Schlepper (zu dieser Figur mehr in Teil 3) bzw. Terrorist als gegenwärtiger Prototyp von Gefahr und Unberechenbarkeit ${ }^{22}$.

Im Gegensatz zu dieser ersten Linie, die sich auf ein globales Außen richtet, zeigt eine zweite, innereuropäisch ausgerichtete, die Pluralität der/des Anderen. Im Widerstreit mit dem politischen Imperativ innerer europäischer Geschlossenheit zeigen sich innereuropäische Grenzen mehr als deutlich - wenn auch typisch begleitet von Forderungen, innereuropäische Spaltung zu überwinden und gemeinsam zu handeln. So wird im folgenden Beispiel eine innere Spaltung Europas angeprangert, die überwunden werden müsse, explizit um mit dem gemeinsamen Problem des illegalen Anderen zurechtzukommen:,,Italiens Innenminister (...) verlangte, die Regeln zu ändern, die die ganze Last der illegalen Einwanderung auf die Länder des ersten Eintritts abwälzten“ (Spiegel, 8.10., auch Kölnische Rundschau, 8.10.). Hier sind innereuropäische Spaltungen zu erkennen, doch das Prinzip europäischer Einheit wird normativ und gerade in Abgrenzung zum globalen Anderen evoziert.

An einem spezifischen Aspekt des hier herangezogenen Diskurses wird jedoch deutlich, dass die Relevanz nationalstaatlicher Grenzen und entlang ihrer verlaufender innereuropäischer Spaltungen sich nicht ohne weiteres in einer gemeinsamen Gegenposition gegenüber einem globalen Anderen aufheben lassen. Konkreter Auslöser ist Friedrichs direkte Verbindung der Dublin-Debatte mit einem innereuropäischen Thema. Er spricht von „Armutseinwanderung (...) von Bulgaren und Rumänen, die angeblich nach Deutschland kommen, um Geld aus den Sozialsystemen zu beantragen" (Spiegel, 8.10.; ähnlich Die Welt, 9.10.). Damit evoziert er ein Bild vom - wohlgemerkt innereuropäischen - kriminellen Anderen und benennt dabei sehr klar zwei europäische Mitgliedsländer, also eigentliche 'Partner'. In den Kommentaren wird genauer hingesehen - und vermeintlich relativiert: „Meist handelt es sich dabei um Angehörige der Roma-Minderheit" (Kölnische Rundschau, 8.10.). Auf diesen Versuch einer Wendung, also das implizite Angebot, ein gemeinsames innereuropäisches Anderes zu konstituieren, gehen die Diskursbeteiligten jedoch nicht ein, wie unten zu sehen sein wird.

Friedrich stellt also eine Art der Wirtschaftskriminalität in den Mittelpunkt: „Friedrich forderte die EU auf, hart dagegen vorzugehen: 'Es kann nicht sein, dass Freizügigkeit so missbraucht wird, dass man ein Land nur deswegen wechselt, weil man höhere Sozialhilfen haben möchte'. Der Minister verlangte, klarzustellen, 'ob wir diejenigen, die zur Leistungserschleichung nach Deutschland kommen, zurückschicken können und ihnen eine Wiedereinreisesperre auferlegen können" (Kölnische Rundschau, 8.10.). Die Forderung, Europa solle sich selbst, vor allem aber Deutschland schützen, wird also auf dem Einsatz von diskursiven Elementen wie Illegalität, Kriminalität und Generalverdacht gegründet. Indem Friedrich auf ein bereits bestehendes innereuropäisches Armuts- und Migrationsproblem verweist, von dem Deutschland vermeintlich überproportional betroffen ist, wehrt er über eine systematische Nutzung des globalen Diskurses und dessen Parallel-

\footnotetext{
${ }^{22}$ So wirft die Gesellschaft für bedrohte Völker Friedrich vor, er habe Ängste geschürt, „unter den tschetschenischen Flüchtlingen befänden sich Terroristen (...). Mit diesem Populismus leiste Friedrich der inhumanen Behandlung vieler Flüchtlinge Vorschub“ (Gesellschaft für bedrohte Völker, Pressemitteilung, 9.10).
} 
führung mit dem europäischen die Ansprüche an Deutschland eben auch auf globaler Ebene ab.

Dass dies den Charakter einer Aufgabenverschiebung an die europäischen Partner hat, erkennen diese, und in ihren distanzierenden Reaktionen werden wiederum verschiedene Befremdungsstrategien sichtbar. Besonders viel Aufmerksamkeit hat in der Presse eine Ironisierung erfahren: „Asylpolitik: EU-Kommissarin kritisiert 'Bierzelt-Aussagen' aus Deutschland“ (Spiegel, 8.10.; ähnlich Kölnische Rundschau, 8.10.). Das exakte Zitat findet sich in der Frankfurter Neuen Presse: „'Der deutsche Minister Friedrich - den kenne ich ja lange - der macht manchmal so Bierzelt-Sprüche. Deshalb mögen ihn ja alle, ich mag ihn auch, und ich mag das Bierzelt auch', spottete EU-Justizkommissarin Reding. Allerdings gehe es um die 'Freizügigkeit von Europas Bürgern', da müsse man schon 'mit Sachargumenten kommen'“ (Frankfurter Neue Presse, 9.10.). Mit dieser Trennlinie, die in diesem Fall quer durch das vermeintliche europäische Selbst führt, wird nicht nur Distanz an sich ausgedrückt. Stattdessen wird diese qualitativ ausgebaut, und zwar durch eine gezielt degradierende Nationalisierung - vor allem gefasst in der Bierzelt-Metapher, die Deutschland zu repräsentieren scheint, die sich isoliert aber durchaus auch als ästhetische Differenzierung begreifen ließe - und eine daraus resultierende Provinzialisierung und Ent-Fremdung Friedrichs.

Schließlich werden auch innerdeutsche Grenzziehungen entlang politischer Positionen deutlich, die allerdings nicht ironisch sondern offen ernsthaft verlaufen (Spiegel, 8.10.; ähnlich Kölnische Rundschau, 8.10.; Zeit online, 11.10.; zur Position der Grünen in dieser Debatte Le Figaro, 9.10.) und auch moralische Abwertungen des politischen Gegners enthalten: „Trittin über Friedrich: 'Abgrund an Zynismus'“ (FAZ, 10.10.). Noch ausgeprägter die Distanz, die im Zitat Riexingers in der Süddeutsche deutlich wird: „Friedrich versucht, die AfD rechts zu überholen. So wird ein Klima erzeugt, das braune Banden ermutigt. Ein Innenminister, der sich als Hassprediger betätigt, ist eine Gefahr für die innere Sicherheit" (Süddeutsche, 9.10., auch FAZ, 8.10. (b)). Hier wird sehr stark auf Differenz abgestellt, indem Vokabular verwendet wird, das im Kontext 'des' gegenwärtigen Anderen des Westens üblich ist, nämlich 'des Islam'. Indem er als gefährlich beschrieben wird, wird versucht, Friedrich als maximal fremd darzustellen. Abgemildert, aber prinzipiell entsprechend, wirkt der Vorwurf von Ulla Jelpke, „der Innenminister werfe 'Nebelkerzen'“ (FAZ, 8.10. (b)). Auch hier haben wir es mit der Unterstellung illegalen Verhaltens zu tun, das als der Gesellschaft schadend, militant gilt. Auf diese Weise wird eine Distanzierung über die Unterstellung von Anderssein und Fremdheit zum Teil überdeutlich symbolisiert - in der Qualität oben beschriebenen Distanzierungs- und Verfremdungsstrategien von Friedrich selbst oft bemerkenswert ähnlich.

Zusammenfassend zeigt sich erstens, dass das globale Andere weitgehend vereinfachend kategorisiert erscheint, während sowohl das europäische als auch das nationale Innen sehr viel stärker differenziert werden. Zweitens manifestieren sich ästhetische Fremdheitsinterpretationen in diesem Diskurs kaum. Das gilt nicht nur für die Fremdheit des globalen, sondern auch des europäischen und besonders des nationalen Anderen. Wenn beispielsweise Italiens damaliger Innenminister von der "ganze(n) Last der illegalen Einwanderung" spricht (Spiegel, 8.10.), oder Malmstroem, damalige EU-Kommissarin für Innenpolitik, fordert, „(a)lle Mitgliedsstaaten ,müssen ihr 'Äußerstes' bei der Aufnahme von Flüchtlingen tun" (Handelsblatt, 8.10.), geht dies über Interpretationen von Anderem als zu Konsumierendem oder als lediglich ästhetisch different deutlich hinaus. Noch deutlicher wird die wahrgenommene bzw. evozierte Gefahr anhand der Beispiele, in denen von grenzüberschreitender Kriminalität, Eindringen des Illegalen etc. gesprochen und das Andere pauschal als gefährlich eingestuft wird. Die Zwischenstufe 'Belastung, nicht Be- 
drohung', die Han im Migrationskontext vorschlägt, findet sich, wenn überhaupt, nur als rhetorisches Mittel im Sinne eines Kleinredens der Sorgen anderer Europäer, um Konsequenzen für das (deutsche) Selbst abzuwenden: „Deutschland ist das Land, das die meisten Flüchtlinge in Europa aufnimmt. Das zeigt, dass die Erzählungen, dass Italien überlastet ist mit Flüchtlingen, nicht stimmen" (Friedrichs in DPA, 8.10., Hervorhebung d.A.). Drittens werden auch innerhalb des europäischen und des nationalen Rahmens essentialistische Definitionen vom Anderen herangezogen. Im europäischen Rahmen wird dies besonders an Friedrichs Einbringen der ,Rumänen und Bulgaren' in die Lampedusadiskussion deutlich. Dabei macht er zwischen ihnen und den aus afrikanischen Ländern stammenden Flüchtlingen keinen Unterschied: Beide werden als fremd und aufgrund ihrer unterstellten kriminellen Potentiale als prinzipiell gefährlich dargestellt. Dieser Diskussionsbeitrag ist besonders interessant, weil er gleichsam auf zwei Ebenen verläuft. Denn mit der Nennung der beiden Länder Bulgarien und Rumänien greift Friedrich zwei europäische Partner an; wie der Kölnischen Rundschau ist aber vermutlich vielen Beobachterinnen und Beobachtern klar, dass Friedrich auf Sinti und Roma anspielt. In den offiziellen politischen Kommentaren wird diese unterschwellige Fremdheitsgrenze jedoch nicht offen aufgegriffen. Stattdessen stabilisieren sie - offenbar gerade ausgelöst durch Friedrichs Parallelführungen - gleichsam das europäische Innen über ihren Protest, der umgekehrt Friedrichs, zwar in verschiedenen Formen, aber übereinstimmend deutlich, mit Anderssein belegt.

\section{Abgrenzung oder Verbindung? Relationierung und Raumkonstruktion}

Wie und unter welchen Bedingungen wirken Grenzen vor allem abgrenzend oder aber stärker angrenzend oder sogar verbindend? Das Argument dieses Abschnitts läuft auf eine Kombination der Aspekte hinaus, genauer: auf das Ausarbeiten der These einer Relationierung, die schon bei Simmel im Vordergrund steht, und die Bedingung der Entstehung von Räumen ist. Damit wird explizit eine Prozessperspektive eingenommen. Mit dem Schwerpunkt bei der Analyse des Materials sei zu diesem Abschnitt lediglich auf zwei interessante Aspekte verwiesen, deren Impuls aus psychoanalytisch inspirierter Forschung stammt.

Sigrun Anselm spricht dem Ort an der Grenze einen eigenen Status zu. Sie schreibt: „Immer hat sich an der Grenze entäußert, was im Innern keinen Platz und keine Artikulationsmöglichkeit hatte (...). An der Grenze finden nicht nur die räumlichen, sondern auch die zivilisatorischen Grenzüberschreitungen statt" (1995, S. 197f.), letzteres Reckwitz' liminalem Grenzaspekt entsprechend. Doch besteht damit zunächst nur ein Ansatz für Relation, und auch ist die Grenze selbst noch nicht zum Raum geworden - die Formulierung , an der Grenze' verweist eher auf eine Angliederung, einen Zusatz, der sich an etwas festzusetzen, anzukoppeln versucht. Davon lässt sich jedoch ein Raumpotential ableiten, das sich durch den impliziten Verweis auf Wiederholungen ergibt, auf die Chance der Entstehung von Typischem, das sich wieder und wieder an Grenze zu ereignen scheint. Diese Kombination aus Praktik(en) und Zeitkomponente bietet ein Potential zu Raumbildung.

Zweitens und in konzeptueller Nähe zu Simmel spezifiziert Anselm Raum als Gemeinsames von Innen und Außen plus Grenze: „Um die eigenen Grenzen finden und sie positiv besetzen zu können, bedarf es eines überschaubaren, organisierten Raums, in Winnicotts Formulierung: des Übergangsraums, der das Innen und Außen umschließt. Das, was außerhalb der Subjektgrenzen liegt, muss selbst erfahrbar, erkennbar und verinnerlichbar sein, so daß das Subjekt sich in einem äußeren Innen situieren kann“ (ebd., S. 200f., Hervorhebung d.A.). Mit anderen Worten: Auch der äußere Raum muss zumindest als relevanter - also in Beziehung zum Innen - erfassbar sein, folglich irgendwelche Ähnlichkei- 
ten und Anknüpfungspunkte bieten, und damit Simmels Mindestkriterium für Vergesellschaftung erfüllen. Entsprechend „müssen die alltäglichen Grenz- und Fremdheitserfahrungen immer wieder über Symbole und Formen assimilierbar sein, die beidem ihr Recht geben: dem Identischen und dem Unterschied. Eine Vielzahl von Verhaltenskodices (...) lassen sich als Synthese zwischen Grenzsetzung und Grenzüberschreitung beschreiben" (ebd., S. 201; Hervorhebung d.A.).

Die Ergebnisse der Diskursanalyse erhellen Raumbildungsprozesse innerhalb der EU gegen die gemeinsame globale Grenze. Insbesondere spezifizieren sie jedoch den Aspekt von Grenze als Raum dazwischen, seine Konstituierung und verschiedenen Elemente, was sich deutlich an Kommentaren zum Grenzraum zwischen der EU und dem Globalen nachzeichnen lässt.

Der erste Raumbildungsaspekt, den Simmel bereits in den ersten Sätzen seines Aufsatzes Der Streit als 'banal' beschreibt, was durch die weite Verbreitung der Vorstellung des 'Sündenbockprinzips' auch prinzipiell bestätigt wird, ist der engere Gruppenzusammenschluss gegen einen als solchen definierten gemeinsamen äußeren Feind. So lässt sich im Innern der EU eine Raumbildungsstrategie erkennen, die sich gerade gegen eine globale Grenze entwickelt und, wie bereits erwähnt, mit einer klaren Trennung - zumindest auf Wahrnehmungsebene - zu arbeiten versucht: einer vermeintlichen Vereinheitlichung nach innen auf der Grundlage einer Abgrenzung nach außen.

An zwei Beispielen lässt sich dieser Prozess nachzeichnen: Der Diskussion um Gerechtigkeit im Innern Europas sowie der um nicht-einheitliche Umsetzung gemeinsamer Regeln der Flüchtlingsbehandlung. Innerhalb der EU besteht erkennbar ein normativer Referenzrahmen, der besagt, dass es eine 'gerechte' Verteilung der Flüchtlinge in der EU geben müsse - also ein Ausgleich innerhalb Europas stattfinden solle, so dass in dieser Hinsicht eine gewisse, den gemeinsamen Raum charakterisierende Homogenität entsteht: „Besonders Italien verlangt, die Aufnahme von Flüchtlingen gerechter zu organisieren“, (Der Spiegel, 8.10.). Österreichs damalige Innenministerin äußerte: „Ich halte sehr viel davon, ein System zu finden, das einfach mehr die Verantwortung gerecht aufteilt" (Die Welt, 8.10., auch Die Welt, 9.10.). Doch lässt sich Gerechtigkeit fraglos ganz unterschiedlich interpretieren: „Für Bundesinnenminister Friedrich ist die Forderung nach mehr Solidarität von Deutschland ,unbegreiflich“" (Süddeutsche, 9.10.), und er „wies auf dem EUInnenministertreffen (...) Forderungen nach einer gerechteren Lastenverteilung in der Flüchtlingspolitik zurück" (Euractiv, 8.10.), und zwar auf der Grundlage von Statistiken. Der zweite Bereich ist der Anspruch einheitlicher Regelanwendung: „Es müsse jetzt zunächst darum gehen, das neue EU-Regelwerk zur Asylpolitik in innerstaatliches Recht zu übertragen und anzuwenden, erläuterte Malmstroem“ (FAZ, 8.10. (a)). Denn bislang hinkt der Ausgleichsprozess, auch aufgrund von zwischen den EU-Staaten "stark variierende(n) (...) Erfolgsquoten“ (Zeit online, 9.10.) bei Asylverfahren. Und gerade das diesbezügliche, sich wiederholende Lamento unterstreicht die Gültigkeit des generellen Anspruchs eines prinzipiell als gerecht betrachteten Ausgleichs, einer generellen Konvergenz im gemeinsamen Raum EU.

Dieser EU-interne Raumbildungsprozess erfährt nur scheinbar einen Bruch durch das Einziehen einer zweiten Ebene, nämlich Friedrichs bereits oben grundsätzlich besprochenen Verweises auf vermeintlich problematische innereuropäische Migration. Erwaehnt sei an dieser Stelle lediglich, dass eine umfassende Gegenrede entsteht und beides gemeinsam gleichsam aufgehoben wird in dem Versuch, mit der Verkündung von gemein- 
samen Zielen einen Interpretationswechsel vorzunehmen. Dazu werden innere Grenzen gegenüber gemeinsamen äußeren und mit ihnen verbundenen Zielen als vergleichsweise unbedeutend definiert - vielleicht nicht als gänzlich ästhetisch, aber ein Versuch des Unsichtbarmachens interner Differenzen lässt sich daran zweifellos erkennen, wenn im Kontext 'der Bulgaren und Rumänen' von einem Europäischen Aktionsplan die Rede ist: „Geld aus dem Sozialfonds soll ab Januar 2014 verstärkt in die soziale Integration und den Kampf gegen Armut gesteckt werden“ (Die Welt, 9.10.).

Die Grenze zwischen der EU und dem Globalen wird auf mehrere Weisen als Raum 'dazwischen' konstituiert. Die sich im untersuchten Diskurs am häufigsten zeigenden Mittel sind durch Mobilität charakterisiert und verdichten sich durch ständige Wiederholungen: Ströme, die Tätigkeit von Schleusern, Boote und Lager.

Im Kontext globalen EU-Grenze wird der erstgenannte Begriff beispielsweise vom EUPräsidenten Martin Schulz benutzt, der sagt, es sei eine „Schande, dass die EU Italien mit dem Flüchtlingsstrom aus Afrika so lange allein gelassen hat" (Der Spiegel, 8.10.). Die Welt spricht von einem „Flüchtlingsandrang aus Afrika“ (8.10.), während sich räumlich genauer die Kölnische Rundschau auf einen „Flüchtlingsstrom() im Mittelmeer" (8.10.) bezieht. Damit werden Naturmetaphern herangezogen, ein Strom, Andrang, gleichsam etwas Flüssiges oder Teilflüssiges vorgestellt, das zum einen nicht fassbar ist und somit besonders unkontrollierbar und bedrohlich wirken kann ${ }^{23}$. Zum anderen wird durch die zeitliche Komponente eines Stroms die Vorstellung eines tendenziell andauernden Prozesses evoziert, was im Prinzip zur Verstetigung der Bewegung an sich und somit zum Grenzraum als relativer Konstante, als eigenem Phänomen führt.

Während Strom und ähnliche Begriffe kollektive Fassungen des Prozesses sind, stellt die Figur des Flüchtlings fraglos die wichtigste individuelle, persönliche, in direktem Sinne auch körperliche Kategorie dieses Raums dar. Hinsichtlich des konstitutiven Prozesses der Raumbildung spielt aber eine andere individuelle Figur eine wichtigere Rolle im analysierten Diskurs, und zwar die des Schleusers bzw. Schleppers ${ }^{24}$. Die Individualisierung des strukturellen Problems gipfelt in einer Erläuterung der Kölnischen Rundschau: „Der Kapitän des vor Lampedusa gesunkenen Flüchtlingsbootes wurde unterdessen in Italien festgenommen. Gegen den 35-jährigen Tunesier wird unter anderem wegen mehrfachen vorsätzlichen Totschlags und Havarie ermittelt" (8.10.). In kollektiver Hinsicht schlägt sich die Kriminalisierung als ausgeprägte Befremdungsstrategie im Begriff 'Schlepperbande' nieder (FAZ, 8.10. (a)), den auch der ORF.at (vom 8.10.) in Form eines Friedrich-Zitats aufgreift: „Wir müssen die Schlepperbanden unschädlich machen“.

Konkret wirkt der Schleuser als Transferermöglicher und -durchführer oder auch Transmitter raumkonstituierend, was durch die ihm unterstellte, fortlaufende Professionalisierung weiter verstärkt wird. Während die Figur des Flüchtlings ambivalent betrachtet wird (dies wird u.a. unten im Kontext der Boote deutlich), gilt der Schleuser nahezu uneingeschränkt als moralisch verwerflich, gefährlich und deshalb zu bekämpfen: „Der Innenminister fordert indes eine härtere Gangart gegenüber Schleppern“ (Euractiv, 8.10.; ähnlich Die Welt, 8.10.). Ein Beispiel, in dem eine Schuld ganz beim Schleuser verortet und der Flüchtling gänzlich zum Opfer wird, ist die Ankündigung von Barakauskas, des damaligen Innenministers Litauens. Er kündigte „einen wirksameren Kampf gegen Menschenhändler an, denen nach Europa strebende Flüchtlinge ausgeliefert seien“ (FAZ, 8.10. (a)). Hier

\footnotetext{
${ }^{23}$ Dass diese spezifische Ausdrucksweise gleichzeitig Wertungen transportiert, wird im Kontrast zum Begriff Migrationsbewegungen deutlich, der für den gleichen raumbildenden Prozess an anderer Stelle genutzt wird (FreitagKommentar, 9.10.).

${ }^{24}$ Auch eine Perspektive auf diese Figur ist die des Grenzyerletzers (siehe z.B. Eigmüller 2008 sowie Horn et al. 2002).
} 
lässt sich der Versuch erkennen, den Schlepper zum gemeinsamen Anderen aller zu machen - und das Signal, mit seinem Ausschalten vermeintlich das Gesamtproblem zu lösen. Die Flüchtlingsorganisation Pro Asyl stellt dagegen fest: „Wer Schleuser bekämpfen will, muss legale Fluchtwege schaffen, nicht die Grenzen abdichten“ (Euractiv, 8.10.). So wird der Grenzraum als solcher weiter manifestiert - gerade durch die Verschiebung der Diskussion auf die Frage des Wie statt auf die des Ob einer Institutionalisierung des Fluchtwegs.

Ein weiteres Bewegungs- und grenzraumkonstituierendes Element ist das Boot. Boote selbst stellen sowohl einen eigenen Raum dar als auch einen zentralen Bestandteil des sich manifestierenden Grenzraums, und zwar einen besonders widersprüchlichen. Sie sind - vor allem besetzt mit Flüchtlingen, aber auch im Besitz der Küstenwache, der Fischer etc. - sein wichtigstes mobiles Transportelement, das 'beide Seiten' systematisch miteinander verbindet. Dabei sind sie zum einen eine kollektive Ausdrucksform der Schleppertätigkeit in dem Sinne, dass nicht nur ihr Überladen, sondern bereits ihr Ablegen an sich als moralisch verwerflich bewertet werden, wie in Teil 1 anhand der AfrikaDiskussion deutlich wurde. Zusätzlich aber enthalten die Boote durch die Beladung mit Flüchtlingen ein Element der Verzweiflung, das die Boote prinzipiell ambivalent wirken lässt, denn es ist offensichtlich, dass für die Flüchtlinge das Boot existentielle Potentiale hat - Leben und Tod. Und dass sie sich dessen bewusst sind, wird vorausgesetzt, sei es explizit oder implizit: „Sie flüchten und viele sterben - doch die Boote kommen weiterhin" (Zeit online, 11.10.).

Seitens der EU werden Flüchtlingsboote grundsätzlich als Objekt von Aktionen interpretiert, die aber relativ unbestimmt und auch widersprüchlich sind. So wird typisch eher allgemein vom „Umgang mit Flüchtlingsbooten auf dem Mittelmeer" (Die Welt, 9.10.) gesprochen. In einer auf schwierigere Weise widersprüchlichen Form zeigt sich diese Ambivalenz in der Beziehung zwischen der EU, nationalstaatlichen Regelungen, Fischern vor der Küste und Flüchtlingen: „Fischern, die Flüchtlinge retten, drohen sogar Geld- oder Haftstrafen. Das hatte (...) dazu geführt, dass Fischer den ertrinkenden Flüchtlingen nicht helfen wollten“ (Zeit online, 9.10.). Und gleichzeitig: „EU-Abgeordnete übten scharfe Kritik an den rigorosen Antiimmigrationsgesetzen, die Fischer mit Strafe bedrohen, sollten sie in Seenot geratenen Flüchtlingen helfen" (ORF.at, 8.10.). Damit werden die Fischer gleichsam in die Rolle der Grenzschützer gezwungen - um sich dann moralisch verurteilt zu sehen.

Ein weiteres konstituierendes Element des Raums Grenze ist das Lager. Im Vergleich mit den gerade genannten Aspekten ist seine Mobilität indirekter zu bestimmen: Sie entsteht gerade aus dem Festhalten der Flüchtlinge in der prinzipiellen Vorläufigkeit ihres Aufenthaltes. Thematisiert werden sie vor allem mit Blick auf Unterbringungsprobleme: „Griechenland, Ungarn und Zypern sperren Neuankömmlinge in völlig überfüllte Lager, in Italien müssen Flüchtlinge oft auf der Straße oder in alten Baracken leben" (Zeit online, 9.10.). Der zweite Teil dieses Zitats verweist auf den zentralen Aspekt, dass die Flüchtlinge nach ihrer Ankunft in Europa in der Regel weiter in einem Zwischenraum gehalten werden, in anhaltenden Transitzonen (ein Paradoxon, denn 'Transit' verweist auf Bewegung), in der Regel sowohl physisch als auch rechtlich und in der Folge psychisch: „Mittlerweile dauern die Zuständigkeitsverfahren nach Dublin meist länger als die eigentlichen Asylverfahren. Das ist teuer, bürokratisch und hält die Flüchtlinge in permanenter Unsicherheit" (Zeit online, 9.10.). Damit wird der Grenzraum, der hier zunächst primär durch das Mittelmeer und die Phase seiner Überquerung bestimmt wurde, durch eine zeitlich deutlich erweiterte Dimension verbreitert. 
Dieser juristischen Grenzraumbildung auf EU-Gebiet geht allerdings eine faktische auf dem Mittelmeer selbst voraus. In den Kommentaren spiegelt sich der Eindruck wider, die EU erweitere einfach ihr Territorium mit der Begründung, die 'anderen' Länder seien nicht in der Lage, zu handeln: „Ein EU-Diplomat kritisierte den Plan als 'naiv', die Lösung sei eine strengere Kontrolle der Küsten Libyens und Tunesiens durch die Küstenwachen dieser Länder" (ORF.at, 8.10.). Und die Kölnische Rundschau zitiert Friedrich: „Boote müssen besser und schneller aufgefunden werden, und es muss dafür gesorgt werden, dass diese Boote in Afrika gar nicht erst ablegen" (8.10.; auch Die Welt, 9.10.). Dies verfestigt gleichzeitig prinzipiell die Grenzen zwischen 'denen' und 'uns', die damit noch die Gelegenheit zum Ausdruck 'unseres Gutmenschentums' haben - im Sinne von 'wir wollen ja helfen'. So wird gerade durch ein vermeintliches Heranrücken ('helfen') eine Distanz verdeutlicht und verfestigt - was zusätzlich als eine moralische Legitimation fortbestehender Unterschiede interpretiert werden kann.

Denn im Vergleich mit dem moralischen Anspruch eines Ausgleichs innerhalb der EU, wie gerade dargestellt, ist ein solcher im globalen Kontext nicht nachzuweisen. Stattdessen wird eine Art von Fairness im gemeinsamen Raum von EU und Globalem gefordert: „Wir müssen jetzt handeln, um ein Gleichgewicht zu finden zwischen unseren Grenzkontrollrechten und den Bedürfnissen und Rechten der Migranten“, betonte FRA-Direktor Morten Kjaerum" (ORF.at, 8.10.). Hier wird das 'faire' Gleichgewicht als Frage der Balance von Abgrenzung (frontier) und Angrenzung bzw. sogar Verbindung (border) behandelt. Dabei unterscheiden sich die Kommentare in ihrer Reichweite: „Nötig wäre die Öffnung der Grenzen. Das mag die Sozialsysteme etwas kosten, aber es geht um mehr: die Menschenwürde" (Freitag-Kommentar, 9.10.).

\section{Verhärtung oder Aufweichung? Ambivalenzen}

Welche Konsequenzen entstehen aus der Verschiebung von Grenze hin zu einem zunehmend offensichtlich differenzierten und differenzierenden, oft ambivalenten sowie tendenziell konfliktuellen Raum? Wie kann Grenze unter diesen Bedingungen ihre grundsätzlichen Funktionen erfüllen, so vor allem die der Komplexitätsreduktion und nicht zuletzt die Legitimierung anhaltender Differenzen?

Dazu lassen sich zwei prinzipielle Einschätzungen vornehmen. Zum einen lässt sich annehmen, dass, wenn bestimmte Grenzen an Bedeutung verlieren - beispielsweise die innerhalb der EU - andere gleichsam als Ausgleich verstärkt werden - z.B. die EUAußengrenzen. Damit werden die Funktionen der Grenze im Grundsatz unverändert gleichsam auf eine zweite Ebene verschoben und bleiben im Prinzip stabil, so dass ihre Funktionalität nicht grundsätzlich in Frage gestellt wird. Eine zweite Interpretation geht dagegen von einem qualitativen Wandel aus, nämlich von der Annahme, dass, wenn einmal das Prinzip klarer Abgrenzung in Frage gestellt ist, sich ein genereller Rechtfertigungsbedarf für jede Grenze ergibt. So stellen Delanty und Rumford auf der Grundlage der Kontrastierung von border und frontier fest: "the outer limit of the EU is becoming more like a border at precisely the same time that national frontiers within the EU are becoming diffuse" (2005, S. 32). Damit leiten die beiden Autoren eine allgemeine Flexibilisierung des Prinzips Grenze ab, das nun grundsätzlich mit entsprechenden, auf Dauer gestellten Legitimationsanforderungen und somit gesteigerten Konfliktpotentialen verbunden ist und offen kontingent wird. Dies macht Grenze prinzipiell angreifbar. Gleichzeitig aber - und damit ursprünglich Simmel folgend - wirkt Konfliktivität selbst aber auch raumbildend, denn es entwickeln sich Chancen zu Sinnverdichtungen und Bedeutungsgeflechten, die Beziehungsbildung begünstigen. Beide Betrachtungsweisen enthalten ein Moment der Unsicherheit, ein mehr oder weniger bewusstes Wahrnehmen von Kontingenz - doch während es in der ersten Interpretation zumindest am Horizont, 
gleichsam nach einer Übergangsphase, eine Rückkehr zur prinzipiell bekannten, klaren Ordnung zu geben scheint, sind in der letzteren Perspektive Kontingenz und ihre Folgen auf Dauer gestellt.

Die in der Einleitung genannte Unterscheidung zwischen analytischer und wahrnehmungsbezogener Perspektive lässt sich hier noch einmal aufgreifen: Im weiteren Sinne ist die erste Interpretation eher der Wahrnehmungsebene zuzuordnen, während die zweite in analytischer Hinsicht interessanter ist. Es wäre jedoch verkürzt, sie als ein bloßes Nebeneinander darzustellen. Tatsächlich wirkt die Wahrnehmungsebene erheblich auf die analytische ein. Schroer nimmt an, dass „Unterscheidungen diffuser werden, ohne gänzlich zu verschwinden. Sie bleiben weiterhin gültig, verlieren aber ihre Exaktheit (...). Und gerade diese Unklarheit provoziert Anstrengungen, wieder zu klaren Grenzziehungen zu kommen. Die Investitionen in eine Linie im Raum zeugen vom großen Bedürfnis nach klaren Trennungen und Unterscheidungen" (2012, S. 180; Hervorhebungen im Original; ähnlich Blaive und Lindenberger 2012, 102) ${ }^{25}$. Entsprechend ist es sinnvoll, statt einer Ablösung des Linienmodells eine analytisch bewusste Parallelführung mit dem Raummodell anzunehmen.

Vor diesem Hintergrund fokussiert der letzte Abschnitt dieser Analyse die sich zeigenden Ambivalenzen des Diskurses, die sich - zumindest bislang - nicht auflösen zu lassen scheinen. Dabei offenbaren sich sowohl inhaltliche Dilemmata, wie beispielsweise die Frage, zu welchem Zweck EU-Schiffe einzusetzen sind, als auch formale, z.B. das gleichzeitige Jonglieren mit Linien- und Rauminterpretationen zur europäisch-globalen Grenze.

Am grundlegendsten ist die Ambivalenz der Grenze selbst. So zeigen beispielsweise Begriffe wie Grenzschutz bzw. in personalisierter Form Grenzschützer (z.B. in DPAPressemitteilung, 8.10.), dass Grenze abstrakt nicht funktioniert, sondern erst durch Diskurs und Handlung wirksam gemacht werden muss. Grundsätzlich ist sie eine gefährdete Zone mit ambivalenten Potentialen.

Der spezielle, hier interessierende Fall des Grenzraums Mittelmeer zwischen Europa und dem Globalen ${ }^{26}$ sowie der Umgang insbesondere mit seinen genannten Elementen 'Flüchtling' und 'Boot' ist ebenfalls prinzipiell ambivalent: „Um Italien direkt zu helfen, stellte die EU mehr Hilfe beim Grenzschutz und der Rettung von Flüchtlingen aus Seenot in Aussicht" (Die Welt, 8.10., ähnlich FAZ, 8.10. (a)). In diesem Beispiel werden zwei regelrecht gegensätzliche Aufgaben unreflektiert zusammengeführt - ganz davon abgesehen, dass hier Italien das Ziel der Hilfe sein soll, nicht die Flüchtlinge. Auf Friedrich Bezug nehmend schreibt das Handelsblatt (8.10.): „Die Grenzsicherungsbehörde Frontex habe in den vergangenen beiden Jahren 40.000 Menschen aus Seenot gerettet und sei damit keineswegs nur ein Instrument der Abschottung, wie immer behauptet werde“. Diesem Paradoxon zugrunde liegen die grundsätzlichen und gegensätzlichen Ziele, einerseits möglichst wenige Flüchtlinge im eigenen Land aufzunehmen, und andererseits Flüchtlinge vor dem Tod zu retten. Dies findet sich nicht offen thematisiert und bleibt somit im Unklaren. Nur in einem Fall wird das Paradoxe der Mittel klar benannt: „Die europäische Grenzschutzagentur Frontex und das Grenzüberwachungsprogramm Eurosur verfügen über die Technik und Ausrüstung, in Seenot geratene Flüchtlinge auf dem Mittelmeer zu entdecken und zu retten. Nur werden sie bisher vor allem zur Abwehr genutzt" (Zeit online, 9.10.).

\footnotetext{
${ }^{25}$ Praktisch lässt sich das an Institutionalisierungsversuchen zur Schaffung je spezifisch begrenzender Ordnung nachvollziehen, aktuell beispielsweise anhand von Pegidabewegungen.

${ }^{26}$ Ähnlich ist jedoch die Situation weiterer Grenzräume rund um die EU oder andere, z.B. dem zwischen Mexiko und den USA.
} 
Stattdessen werden in der Regel ambivalente Lösungsansätze vorgeschlagen. Eine Strategie ist, das Problem durch Aufspaltung zu lösen. So fordert Friedrich eine Kombination aus Maßnahmen, die an ganz verschiedenen Punkten ansetzen: Verbesserung der Seenotrettung, Verbesserung der Lage der Heimatländer der Flüchtlinge und härteres Vorgehen gegen Schleuser (Die Welt, 8.10.).

Eine zweite Strategie ist eine Technisierung des Themas bzw. des nun scheinbar um das gesamte Mittelmeer erweiterten Grenzraumes, beispielsweise über Eurosur - allerdings zeigt schon diese Namensgebung den Fokus der Maßnahme an: „Das System soll mit Hilfe von Satellitentechnik, Überwachungsdrohnen und einem intensiveren Informationsaustausch zwischen den Mitgliedsstaaten die Außengrenzen der EU sicherer machen" (Euractiv, 8.10.). Dies ist ein offensichtlich militärisch angelehnter Ansatz. Ein medizinisches Prinzip spiegelt sich dagegen im wiederholten Gebrauch des Begriffes 'Operation' wider (z.B. FAZ, 8.10. (a); Handelsblatt, 8.10.), sowie im folgenden Zitat: „Die EU will Italien (...) auch mit einer Task Force unter die Arme greifen“, einer 'Expertengruppe' (Kölnische Rundschau, 8.10.). Diese Strategie der Technisierung evoziert die Vorstellung, dass es faktisch einen richtigen Weg gibt, mit dem Problem umzugehen. Dieser müsse lediglich von Fachleuten sachverständig, einer medizinischen Operation gleich, durchgeführt werden, dann sei das Ganze eine 'saubere Sache'. Im weiteren Sinne gehört zu diesem Ansatz auch das Argumentieren mit jeweils 'passenden' Statistiken, wie oben im Kontext der Gerechtigkeitsdiskussion beispielhaft beschrieben.

Schließlich finden sich vereinzelt auch zumindest auf den ersten Blick konsequenter erscheinende Fragen und Kommentare, nämlich dazu, ob die Grenzen vollständig zu öffnen seien: „Bosbach sagte (...): '(...) Sollen die Grenzen geöffnet werden für alle, die kommen wollen, oder nicht?' Wer für offene Grenzen plädiere, müsse der Bevölkerung sagen, welche Konsequenzen das haben werde" (FAZ, 8.10. (b)). Mit einer solchen zwar umfassenden, letztlich aber rhetorischen Infragestellung der Grenze wird die vermeintlich offene Diskussion jedoch zumindest zunächst tendenziell auf zwei Alternativen reduziert: auf Abwehr oder aber Überlaufenwerden.

Dass es keine klare Lösung gibt, die ein für allemal stehen kann, ist wohl allen Beteiligten klar. Doch das Eingeständnis, keine Lösung für das Problem zu haben, ist zumindest in diesem Material ein Einzelfall: Die EU, „Friedensnobelpreisträgerin des Jahres 2012, stößt bei der vielbeschworenen Solidarität in puncto Flüchtlinge an ihre Grenzen“ (Der Spiegel, 8.10.), innere Grenzen manifestieren sich also als äußere. Und so werden Kompromissvorschläge, wenden sie sich denn überhaupt der substanziellen Frage der Menschenwürde zu (siehe auch Teil 3), auffallend schwammig, zum Beispiel, wenn Konzepte für eine „humanere Aufnahmepolitik“ (Zeit online, 9.10.; Hervorhebung d.A.) vorgeschlagen werden. Denn genau an diesem Punkt gerät die Unterscheidung von Selbst und Anderem substanziell ins Wanken und mit ihr unumgänglich das Konzept der Grenze. Und dennoch: Darin ließe sich durchaus ein Verweis auf die Chance anhaltender Kommunikation sehen - und entsprechender Vergesellschaftungspotentiale.

\section{Fazit}

Unabhängig von der jeweils betrachteten Ebene hat sich Grenze als Kommunikationsanlass und genauer: als Anlass zu (konfliktiver) Auseinandersetzung gezeigt. Das Textmaterial offenbart ganz deutlich ihr typisch zuspitzendes Wirken, das gleichzeitig an dem Prinzip des Kontrasts ausgerichtet und auf irgendeine Art von Ausgleich (Inner-EU-Grenzen) oder zumindest Fairness (EU-globale Grenze) angelegt ist. So haben sich Grenzräume und ihre vielfältigen Entstehungsaspekte - also Praktiken, materielle Manifestationen sowie spezifische Logiken und auch Ambivalenzen - auf verschiedenen Ebenen nachzeichnen lassen, einschließlich Formen des Konzepts Niemandsland. Auch Verfestigungen in For- 
men von Institutionalisierung sind erkennbar (z.B. Eurosur, Pegida). In diesem Sinne muss Durkheim und Parsons zwar nicht unbedingt darin zugestimmt werden, dass das Grenzformat Linie dem des Raumes vorangehen muss, aber da Linie grundsätzlich konfliktträchtig ist, schafft sie, zumindest nach Simmel, als Prinzip von Abgrenzung und Anbindung mit hoher Wahrscheinlichkeit Bedingungen für Vergesellschaftung und somit für entsprechende Raumbildung: Sowohl nach innen (EU) als auch in Wechselwirkungen mit dem globalen Außen verdichtet sich gemeinsamer Sinn, entwickeln sich Bedeutungsgeflechte, Beziehungen und Asymmetrien.

Selbste und Andere haben sich differenziert dargestellt. Dabei sind Selbste in der Regel von vornherein als in sich differenzierter angenommen worden, während beim Anderen viel stärker Simplifizierungen (Afrika) und zusätzliche Verfremdungen, oft über Kriminalisierung, vorgenommen wurden. Umgekehrt waren kaum Differenzierungen der Flüchtlinge zu erkennen. Dass sich aber Selbste und Andere im Wandel befinden, wurde fraglos an Verweisen auf Humanität und Menschenwürde deutlich, die letztlich eine klare, linienförmige Selbst-Fremd-Grenze grundlegend in Frage stellen.

Damit wird die Kontingenz von Anderen, Selbsten und Grenzen gesteigert, was zu Vereinfachungen führen konnte (z.B. Kriminalisierung, Technisierung von Lösungen), aber nicht musste. Im Gegenteil wurde an einigen Textbeispielen erkennbar, dass Kompromisse gesucht ('humaner'), Paradoxa benannt (Einsatz von Frontex und Eurosur) oder auch nur relativ hilflos Ambivalenzen dargestellt wurden ('Es können nicht alle kommen'). Damit ist prinzipiell die Annahme von Delanty und Rumford bestätigt, dass sämtliche Grenzen (zumindest im hier untersuchten Kontext) nun mit grundsätzlichen, vermutlich auf Dauer gestellten Legitimationsanforderungen belegt sind. Auf dieser Grundlage sind entsprechend nicht eindimensionale Verfestigungen zu erwarten, i.S. einer unzweideutigen 'Rückkehr' zu nicht hinterfragbaren Selbsten, Anderen und entsprechenden Grenzen. Gesteigerte Konflikt- und Vergesellschaftungspotentiale scheinen eher in Richtung einer Pluralisierung, Diversifizierung und Ausbreitung von Grenzdiskursen zu verweisen: Wie Schallwellen stoßen globale Ereignisse Diskurse auf allen möglichen Ebenen an - und verbinden diese somit letztlich mit dem Globalen. Es scheint, dass dabei besonders die typischen und fortbestehenden Paradoxa zu tiefgehenden Aussagen über 'uns', 'die anderen' und unsere Beziehungen führen.

\section{QUELLEN}

\section{Literatur}

Anselm, S. (1995). Grenzen trennen, Grenzen verbinden. In R. Faber \& B. Naumann (Hrsg.), Literatur der Grenze - Theorie der Grenze (S. 197-209). Würzburg: Königshausen \& Neumann.

Augé, M. (1995). Krise der Identität oder Krise des Andersseins? Die Beziehung zum Anderen in Europa. In W. Kaschuba (Hrsg.), Kulturen - Identitäten - Diskurse: Perspektiven europäischer Ethnologie (S. 85-99). Berlin: Akademie Verlag.

Bach, M. (2008). Europa ohne Gesellschaft. Politische Soziologie der Europäischen Integration. Wiesbaden: Westdeutscher Verlag.

Balibar, É. (2004). We the People of Europe: Reflections on Transnational Citizenship. Princeton, NJ: Princeton University Press.

Beck, U. (1997). Was ist Globalisierung? Irrtümer des Globalismus - Antworten auf Globalisierung. Frankfurt a.M.: Suhrkamp. 
Blaive, M. \& Lindenberger, Th. (2012). Border Guarding as Social Practice. A Case Study of Czech Communist Governance and Hidden Transcripts. In M. Silberman, K. E. Till \& J. Ward (Hrsg.), Walls, Borders, Boundaries. Spatial and Cultural Practices in Europe (S. 97-112). New York: Berghahn Books.

Bös, M. (2001). Zur Kongruenz sozialer Grenzen. Das Spannungsfeld von Territorien, Bevölkerungen und Kulturen in Europa. In M. Bach (Hrsg.), Die Europäisierung nationaler Gesellschaften (S. 429-455). Sonderheft der Kölner Zeitschrift für Soziologie und Sozialpsychologie 40. Wiesbaden: VS.

Delanty, G. \& Rumford, Ch. (2005). Rethinking Europe. Social theory and the implications of Europeanization. London / New York: Routledge.

Donnan, H. \& Wilson, Th. (1999). Borders: Frontiers of identity, nation and state. Oxford: Berg.

Durkheim, É. (1984). Die elementaren Formen des religiösen Lebens. Frankfurt a.M.: Suhrkamp.

Eigmüller, M. (2006). Der duale Charakter der Grenze. Bedingungen einer aktuellen Grenztheorie. In ders. \& G. Vobruba (Hrsg.), Grenzsoziologie. Die politische Strukturierung des Raumes (S. 55-74). Wiesbaden: VS.

Eigmüller, M. (2008). Subversionen an Staatsgrenzen - eine Einleitung. In dies. \& A. Müller (Hrsg.), Subversionen am Rande. Grenzverletzungen im 20. und 21. Jahrhun$\operatorname{dert}($ S. 13-22). Leipzig: Leipziger Universitätsverlag.

Eigmüller, M. \& Vobruba, G. (2006). Einleitung: Warum eine Soziologie der Grenze? In dies. (Hrsg.), Grenzsoziologie. Die politische Strukturierung des Raumes (S. 7-11). Wiesbaden: VS.

Esposito, R. (2004). Immunitas. Schutz und Negation des Lebens. Zürich: Diaphanes.

Gifford, P. (2010). Defining 'Others': How Interperceptions shape Identities. In ders. \& T. Hauswedell (Hrsg.), Europe and its Others. Essays on Interperception and Identity (S. 13-38). Bern: Peter Lang.

Han, B.-C. (2014). Müdigkeitsgesellschaft. Berlin: Matthes \& Seitz.

Horn, E., Kaufmann, S. \& Bröckling, U. (2002). Grenzverletzer. Von Schmugglern, Spionen und anderen subversiven Gestalten. Berlin: Kulturverlag Kadmos.

Karafillidis, A. (2010). Grenzen und Relationen. In J. Fuhse \& S. Mützel (Hrsg.), Relationale Soziologie. Zur kulturellen Wende der Netzwerkforschung (S. 69-95). Wiesbaden: VS.

Kristeva, J. (1988). Etrangers à nous-mêmes. Paris: Fayard.

Krossa, A. S. (2013). Theorizing Society in a Global Context. Europe in a Global Context Series. Houndmills, Basingstoke: Palgrave Macmillan.

Langenohl, A. (2010). Imaginäre Grenzen. Zur Entstehung impliziter Kollektivität in EUEuropa. In Berliner Journal für Soziologie 20/1, S. 45-63.

Langenohl, A. (2015). Town Twinning, Transnational Connections and Trans-local Citizenship Practices in Europe. Europe in a Global Context Series. Houndmills, Basingstoke: Palgrave Macmillan.

Löw, M. (2012). Raumsoziologie. Frankfurt a.M.: Suhrkamp.

Matzinger, P. (2007). Friendly and dangerous signals: is the tissue in control? In Nature Immunology 8/1, S. 11-13.

Medick, H. (2006). Grenzziehungen und die Herstellung des politisch-sozialen Raumes. Zur Begriffsgeschichte und politischen Sozialgeschichte der Grenzen in der Frühen Neuzeit. In M. Eigmüller \& G. Vobruba (Hrsg.), Grenzsoziologie. Die politische Strukturierung des Raumes (S. 37-51). Wiesbaden: VS.

Perera, S. (2007). A Pacific Zone: (In)security, Sovereignty, and Stories of the Pacific Borderscape. In P. Kumar Rajaram \& C. Grundy-Warr (Hrsg.), Borderscapes: Hidden 
Geographies and Politics at Territory's Edge (S. 201-227). University of Minnesota Press: Minneapolis.

Parsons, T. (1966). Societies. Evolutionary and Comparative Perspectives. Englewood Cliffs/NJ: Prentice-Hall.

Pries, L. (2008). Die Transnationalisierung der sozialen Welt. Frankfurt: Suhrkamp.

Pries, L. (2010). Transnationalisierung. Theorie und Empirie grenzüberschreitender Vergesellschaftung. Wiesbaden: VS.

Ray, L. (2007): Globalization and Everyday Life. London / New York: Routledge.

Reckwitz, A. (2008). Unscharfe Grenzen. Perspektiven der Kultursoziologie. Bielefeld: transcript.

Rigo, E. (2006). Trafficking Citizenship. Von der ,Festung Europa' zur Regierung der Zirkulation'. In Th. Hengartner \& J. Moser (Hrsg.): Grenzen und Differenzen. Zur Macht sozialer und kultureller Grenzziehungen (S. 161-174). Leipzig: Leipziger Universitätsverlag.

Sassen, S. (2013). Analytic Borderlands: Economy and Culture in the Global City. In G. Bridge \& S. Watson (Hrsg.), The New Blackwell Companion to the City (S. 210-220). Malden: Wiley Blackwell.

Schroer, M. (2012). Räume, Orte, Grenzen. Auf dem Weg zu einer Soziologie des Raums. Frankfurt a.M.: Suhrkamp.

Simmel, G. (1992). Soziologie. Untersuchungen über die Formen der Vergesellschaftung. Frankfurt a.M.: Suhrkamp.

Strüver, A. (2008). Bildbesprechung. Das Mittelmeer als Grenzdiskurs. In H. Bredekamp, M. Bruhn \& G. Werner (Hrsg.): Bildwelten des Wissens. Kunsthistorisches Jahrbuch für Bildkritik (S. 66-69). Band 6/2, Grenzbilder. Berlin: Akademie-Verlag.

Vobruba, G. (2005). Die Dynamik Europas. Wiesbaden: VS.

Wilson, Th. M. (2012). Revisiting the Anthropology of Policy and Borders in Europe. In D. Andersen, M. Klatt \& M. Sandberg (Hrsg.), The Border Multiple. The Practicing of Borders between Public Policy and Everyday Life in a Re-Scaling Europe (S. 77-95). Farnham, Surrey: Ashgate.

\section{Textmaterial zur Diskursanalyse}

8.10.2013:

Der Spiegel: Asylpolitik: EU-Kommissarin kritisiert ,Bierzelt-Aussagen' aus Deutschland Die Welt: Illegale Einwanderer. EU kündigt Großeinsatz beim Grenzschutz an

DPA: EU will Flüchtlingspolitik nicht ändern

Euractiv: Friedrich: Kein Anlass für Neuausrichtung der Flüchtlingspolitik

FAZ (a): Katastrophe vor Lampedusa: EU uneins über Flüchtlinge

FAZ (b): Streit über Flüchtlinge: Riexinger nennt Friedrich ,Hassprediger'

Handelsblatt: Friedrich will Flüchtlingspolitik beibehalten

Kölnische Rundschau: Kritik an Friedrichs ,Bierzelt-Aussagen“

ORF.at: Alte Regelungen bleiben

The Guardian: EU pressed to rethink immigration policy after Lampedusa tragedy

9.10.2013:

Die Welt: Flüchtlingspolitik unverändert

Frankfurter Neue Presse: Spott für Friedrichs ,Bierzelt-Aussagen`

Freitag-Kommentar: Ein zynischer Minister

Gesellschaft für bedrohte Völker: Versachlichung der Flüchtlingsdiskussion unbedingt notwendig GfbV warnt Innenminister Friedrich vor Populismus

Le Figaro: Réfugiés: Merkel attaquée par les Verts 
Süddeutsche: Unglück vor Lampedusa: Friedrich sieht keinen Änderungsbedarf bei Asylpolitik

Zeit online: Lampedusa-Katastrophe: Europa kann den Flüchtlingen helfen 10.10.2013:

FAZ: Schwarz-grüne Sondierung: Trittin über Friedrich: ,Abgrund an Zynismus‘ 11.10.2013:

Zeit online: Flickwerk Flüchtlingspolitik 